\title{
Berichtigung
}

zur Arbeit

\section{Zur Frage der körperlichen Entwicklung bei angeborenen Herzfehlern}

von

\author{
A. Sohaede, H. Lo'tzkes und H. H. Hilger
}

Arch. Kreislaufforschg. 24, 1-26 (1956).

Infolge Änderung der Reihenfolge der Abbildungen sind in der oben bezeichneten Arbeit mehrere Legenden in ihrer Zuordnung zu den Abbildungen vertauscht worden, sowie Fehlzitierungen von Abbildungen im Text unterlaufen. Im folgenden wird die richtige Zugehörigkeit aufgeführt.

\section{Legendensuordnung:}

Abb. 4 Legende bei Abb. 12, S. 19 unten (dort bezeichnet als "Abb. 12“)

Abb. 5 Legende bei Abb. 13, S. 20 oben (dort bezeichnet als „Abb. 13“)

Abb. 6 Legende bei Abb. 14, S. 20 unten (dort bezeichnet als „Abb. 14“ und als ,Legende zu den Abb. 12-14"; richtig: Legende zu den Abbildungen 4-6")

Abb. 7-10 bei Abb. 7 = richtig, jedoch fehlerhaft bezeichnet als ,Abb. 4-7“ und als „Legende zu den Abb. 4-7“; richtig: „Abb. 7-10“ und „Legende zu den Abb. 7-10\% 
Abb. 11-14 bei Abb. 11 = richtig, jedoch fehlerhaft bezeichnet als ,Abb. 8-11“ und als ,Legende zu den Abb. 8-11; richtig: „Abb. 11-14“ und „Legende zu den Abb. 11-14:*

Entsprechend sind die Zitierungen im Text zu ändern.

\section{Sonstige Textkorrekturen:}

Seite 3, Zeile 19 v. unt.: ... normal $90 \mathrm{~mm} \mathrm{Hg}$ (statt $900 \mathrm{~mm} \mathrm{Hg}$ )

Zeile 9, Zeile 13 v. unt.: ... Abb. 1 (503, ,Geigy-Fälle“) statt 503 Fälle 